Em Portugal, a investigação em Química teve um grande progresso nestas duas últimas décadas. Houve investimento em meios humanos e materiais, a que a ajuda da Comunidade Europeia deu alento. Mas a hora da verdade está a aproximar-se. Está a chegar o momento em que vamos ficar entregues às nossas próprias forças. E como a economia está mal, o dinheiro é pouco. Mas entretanto as solicitações são múltiplas.

Por um lado, cabe aos Governos tomar as decisões que melhor sirvam o desenvolvimento. $E$ vai ser preciso melhorar o presente e acautelar o futuro. 0 património científico de um País leva muito, muito tempo mesmo, para crescer e tornar-se adulto. E, qual árvore, pode morrer rapidamente se deixar de chover. Por outro lado, os investigadores portugueses têm de pensar em medidas que possam rentabilizar os recursos. Não vai ser possivel continuar a multiplicar equipamentos caros. Não vai ser possivel haver investigadores, donos absolutos de aparelhos. É evidente que se pode correr o risco de ter uns tantos a manter a aparelhagem para outros se limitarem a ser colectores. É um risco. E, por tal, é pre- ciso haver, ao mesmo tempo, um poder bem estruturado, legítimo, sensivel e justo. Sempre que possível, parece-nos desejável haver serviços comuns geridos por técnicos competentes, e aos quais os investigadores possam recorrer. A nosso ver, a palavra de ordem deveria ser organização. Até agora temos visto cada um a puxar a carroça por si. De certo modo, terá de continuar a ser assim, porque a nvestigação também vive da competição. Esta estimula e educa as vontades. $E$ todos nós temos de dar provas todos os dias. Até que o sopro vital se apague. Mas a competição não anula, antes pelo contrário, pode até estimular a colaboraçẵo. Podemos e devemos ser inteligentes e ter uma organização onde todos possam vir a ganhar. $E$ guardar as energias assim libertadas para as grandes causas, as tais que são a essência da descoberta, as que permitem usufruir da beleza da Química. E, esperamos que muitas vezes, criar as condições para descortinar nos nossos alunos esse mesmo prazer. Sejam alunos do secundário, sejam do superior. Porque a descoberta, ou mesmo a revelação, são momentos únicos.
Uma nota final. A sociedade moderna não tem Química a mais, tem é Química a menos. É preciso melhorar a produção, consumindo menos energia, poluindo cada vez menos e limpando cada vez mais. E essa é uma tarefa que os Químicos sabem fazer bem. Entre tantas outras. E se o projecto de revisão dos curricula do secundário for avante, a Química em Portugal vai ficar mais pobre e isso não vai ser bom para o nosso País. Será que vai chegar o dia em que vamos importar os químicos, e principalmente o conhecimento da Química, dos nossos irmãos de Espanha ou de outro lado qualquer? Não é que daí venha mal ao mundo, mas a troca é um bem precioso, se todos os intervenientes tiverem algo para dar. Uma troca só a receber, não é troca, é algo como uma transfusão de sangue. Que recebe só quem está muito doente. Sem pessimismos, porque a vontade de fazer melhor pode mudar a realidade, a todos desejamos uma boa leitura deste vosso boletim. Motivos de interesse não faltam. Desde a entrevista ao Prof. Júlio Pedrosa, ex-Reitor de Aveiro, ex-Ministro da Educação, mas primeiro que tudo um Químico, às secções habituais.

\section{Carteira de Conferências para o Ensino Secundário}

Respondendo a solicitações de vários sócios, a direcção da SPQ decidiu criar uma "Carteira de Conferências" destinada a públicos variados do ensino secundário.

Este projecto teve a adesão (entusiástica, nalguns casos) de um conjunto de docentes que permitiram a formação do núcleo inicial da carteira: uma lista de 22 temas, publicada neste número do
Boletim e divulgada no sítio da SPQ (www.spq.pt)

Os professores interessados em levar à sua Escola qualquer destas conferências podem agcra contactar directamente com o conferencista escolhido e acordar as condições necessárias (evidentemente, a adesão à carteira indica a disponibilidade mas não a obrigatoriedade de satisfazer todas as solicitações). Para facilitar a escolha das conferências, a "Carteira" inclui, além do tema, algumas informações relativas ao público-alvo, e ao equipamento a fornecer pela Escola (para além do vulgar retroprojector)

Ao lançar a "Carteira", a SPQ está a dar início a mais uma actividade que pretende ir ao encontro dos interesses e necessidades dos sócios. Só que "dar início" de nada serve se ninguém estiver disposto a "dar continuidade"... A base de partida - 22 temas - é um começo, mas não pode ser tudo e esperam-se novas adesōes. Em particular, dado o âmbito predominantemente regiona 
preferido pelos conferencistas, esperase que as Delegações Regionais mostrem empenho em garantir aos seus sócios uma "Carteira" diversificada e interessante. Aliás, talvez seja possivel promover uma competição entre Delegações Regionais, cujo dinamismo seria avaliado pelo número de conferências na "Carteira"! À partida, a Delegação Regional de Aveiro leva alguma vantagem...

Novas propostas de temas para a "Carteira" poderão ser enviadas para os presidentes das Delegações Regionais ou directamente para pclaro@dq.ua.pt.

\author{
A. J. Ferrer Correia - DQ-UA \\ (ferrer@dq.ua.pt) \\ A Química e a Côr \\ Conferência; $10^{\circ}$ - $12^{\circ}$ ano; \\ António Lopes - ITQB - UNL \\ (alopes@itqb.unl.pt) \\ Química (e outras ciências) na Cozinha \\ Conferência; $10^{\circ}$ - $12^{\circ}$ ano;
}

António Lopes - ITQB - UNL

(alopes@itqb.unl.pt)

Química dos detergentes e detergência

(Química Coloidal)

Conferência; $10^{\circ}-12^{\circ}$ ano;

Diana Cláudia Pinto - DQ - UA

(diana@dq.ua.pt)

Química Orgânica na melhoria das condições de vida do Homem

Conferência; $10^{\circ}-12^{\circ}$ ano;

Eduarda Pereira - DQ - UA

(eduper@dq.ua.pt)

A ria de Aveiro: detectar e explicar fe-

nómenos de poluição

Conferência; $10^{\circ}$ - $12^{\circ}$ ano;

Hugh D. Burrows - DQ - UC

(burrows@ ci.uc.pt)

Química e Luz

Palestra; $9^{\circ}-12^{\circ}$ ano;

Isabel Boal - DQ - UA

(iboal@dq.ua.pt)

Determinação de $\mathrm{pH}$ - como e porquê?

Conferência; $10^{\circ}-12^{\circ}$ ano;
João Coutinho - DQ - UA

(jcoutinho@dq.ua.pt)

A Engenharia Química e as outras artes

Conferência; $10^{\circ}$ - $12^{\circ}$ ano;

José Carlos Marques - DQ - UMa

(marques@uma.pt)

O Impacto ambiental e a Sociedade

O ambiente e o impacto resultante da

actividade humana

Conferência; $10^{\circ}$ - $12^{\circ}$ ano; Requer

Projector LCD

M. Graça Marques - DQ - UA

(grmarques@dq.ua.pt)

Espectrometria de Massa : dos isótopos às biomoléculas

Conferência; $12^{\circ}$ ano;

M. J. E. Prieto - IST - UTL

(prieto@ist.utl.pt)

Luminescência atómica e molecular

Conferência; $10^{\circ}-12^{\circ}$ ano;

M. N. Berberan e Santos - IST - UTL (berberan@ist.utl.pt)

Luminescência atómica e molecular

Conferência; $11^{\circ}-12^{\circ}$ ano;

Maria Clara Ferreira Magalhães - DQ UA (mclara@dq.ua.pt)

A Química no Ambiente

Conversas com os alunos; $5^{\circ}-12^{\circ}$ ano;

Maria Clara Ferreira Magalhães - DQ UA (mclara@dq.ua.pt)

\section{A Química no dia a dia}

Conversas com os alunos; $6^{\circ}-12^{\circ}$ ano;

Maria do Amparo Faustino - DQ - UA

(faustino@dq.ua.pt)

Na rota de novos compostos orgânicos com actividade biológica

Conferência; $10^{\circ}-12^{\circ}$ ano;

Paulo Ribeiro Claro - DQ - UA

(pclaro@dq.ua.pt)

Porque é que o gelo funde a zero graus?

(\& outras perguntas acerca das pro-

priedades da água)

Conferência; $10^{\circ}-12^{\circ}$ ano;
Rui M. M. Brito - DQ - UC

(brito@ci.uc.pt)

Doenças Amilóides: Que culpas para a

Termodinâmica e a Cinética?

Traços comuns às Doenças dos Pezi-

nhos, de Alzheimer's e das Vacas Lou-

cas

Palestra; $10^{\circ}-12^{\circ}$ ano; Requer Datashow

Sebastião Formosinho - DQ - UC

(sformosinho@qui.uc.pt)

Co-incineração: Um caso Exemplar

Palestra; $9^{\circ}-12^{\circ}$ ano; Requer Datashow

Sebastião Formosinho - DQ-UC (sformosinho@qui.uc.pt)

A química nos dias de hoje

Palestra; $9^{\circ}-12^{\circ}$ ano; Requer Datashow

Teresa Margarida dos Santos - DQ -

UA (teresa@dq.ua.pt)

Compostos com Metais: aplicaçōes em

Medicina

Conferência; $9^{\circ}-12^{\circ}$ ano;

Tito Trindade - DQ - UA

(ttrindade@dq.ua.pt)

Nanomateriais:

Pequenas peças para um grande palco Conferência; $10^{\circ}-12^{\circ}$ ano; Requer Data-show

Vitor Manuel Matos Lobo - DQ - UC (vlobo@ci.uc.pt)

A necessidade de exames no ensino básico e secundário

Conversa/Debate; $9^{\circ}$ - $12^{\circ}$ ano;

DQ - Departamento de Química; ITQB - Instituto de Tecnologia Química e Biológica;

UA - Universidade de Aveiro;

UMa - Universidade da Madeira;

UC - Universidade de Coimbra;

UNL - Universidade Nova de Lisboa;

UTL - Universidade Técnica de Lisboa. 


\section{Olimpíadas de Química 2003}

Estão aí de novo! Com um crescente envolvimento das Escolas - e, particularmente, dos professores da área Disciplinar de Química e de Física e Química as Olimpíadas de Química já garantiram a sua posição entre as actividades de maior sucesso da $\mathrm{SPQ}$.

Este ano inscreveram-se nas semifinais 86 equipas, o que representa um aumento de 30\% em relação a 2002 . Aliás, desde a primeira edição em 2000 que o número de participantes não pára de aumentar (ver gráfico).

A data de publicação deste Boletim terão já decorrido as semifinais, e a lista dos 27 finalistas deverá estar disponivel na página das Olimpíadas em www.spq.pt. A 2 de Maio decorrerá a prova final, que vai apurar os medalhados de 2003 e seriar os candidatos a

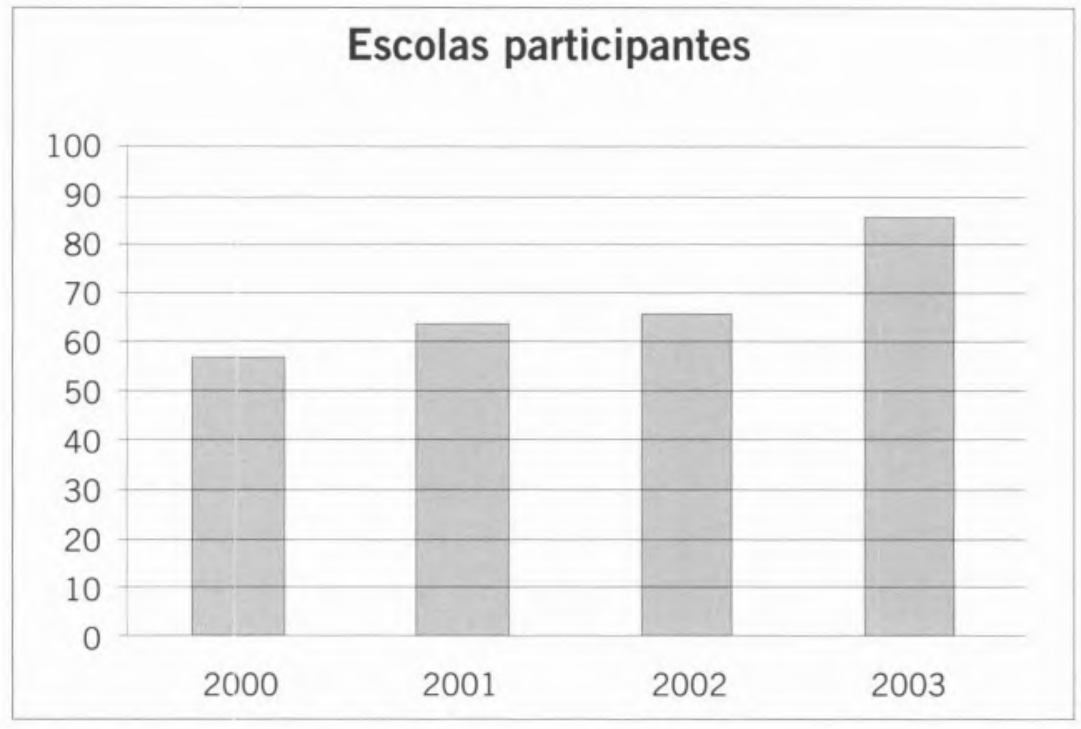

duas viagens aliciantes: as "Olimpiadas de Química 2003" marcam o início da participação de Portugal nas Olimpíadas Internacionais de Química (a realizar em Julho, na Grécia), mantendo a participa- ção nas Olimpíadas Ibero-americanas (a realizar em Outubro, no México). Mais um prémio de participação, a somar àqueles que são distribuídos a professores e alunos!

\section{Mariana Pereira (1950-2003)}

Mariana Pereira faleceu no passado dia 8 de Fevereiro, após prolongada doença, com a qual lutou corajosamente durante muito tempo. Contava apenas 53 anos.

Com uma formação inicial em Química, especializou-se na área de Educação, tendo feito o Mestrado em Educação em Ciências em 1975 em Evanston, nos Estados Unidos, na Northwestern University. Em 1981 obteve o grau de Doutor em Química - Educação em Química, na Universidade de East Anglia, Norwich, no Reino Unido. Era Professora Catedrática do Departamento de Educação da Faculdade de Ciências de Lisboa, desde a sua formação em 1983.

A sua vida foi dedicada ao ensino e à investigação em Educação em Química e em Ciências. Nos anos iniciais da sua carreira leccionou em Escolas Secundárias. Apesar de ter depois transitado para o Ensino Superior, manteve sempre uma grande ligação a escolas de outros graus de ensino, quer na orientação de Estágios Pedagógicos quer em Projectos de Investigação e Desenvolvimento Curricular, nacionais e internacionais. Entre estes são de destacar vários projectos no âmbito do "Ciência Viva" e a coordenação nacional do projecto "Science Accross Europe" em que era também membro da equipa internacional de desenvolv mento curricular.

Profundamente interessada em fomentar a participação dos alunos na realização de trabalho experimental nas escolas e conhecedora das dificuldades ai encontradas, preconizava a utilização de material em pequena escala que poderia ajudar a ultrapassar essas dificuldades. Nesse sentido, apresentava e explorava, com os seus alunos de mestrado ou na formação inicial e em serviço de professores, experiências de química com diversos tipos de material em pequena escala e preferencialmente de baixo custo.

As questões relativas à Educação Ambiental e mais recentemente à Educação para a Sustentabilidade também eram alvo de um seu grande interesse. Nos últimos anos a História da Química e do seu ensino foram novos tópicos a que dedicou parte da sua atenção e do seu tempo.

Era membro activo de diversas sociedades científicas nacionais e internacionais, tais como ACTD, ASE, DIRES, IOSTE, NAAEE, RIEEE, SPCE, SPF, SPQ, WAER/AMSE.

Organizou e participou activamente em muitas conferências e reuniões em Portugal e em muitos outros países, apresentando conferências plenárias, comunicaçōes e workshops. Deixa uma longa lista de publicaçōes (livros, artigos em revistas e actas e CDs).

Como sócia da Sociedade Portuguesa de Química foi particularmente activa. Durante alguns anos, dinamizou e na prática promoveu a organização das provas semifinais da região sul das Olimpíadas de Química, que tinham carácter experimental e chegavam a envolver trabalho laboratorial de mais de uma centena de alunos de diversos ní- 
veis - do $8^{\circ} \mathrm{ao} 12^{\circ}$. Editou também e traduziu parcialmente vários números da versão portuguesa da International Newsletter on Chemical Education da IUPAC.

\section{Encontro Nacional de Química Orgânica}

\section{Universidade de Aveiro, 1 a 4 de Julho de 2003}

$05^{\circ}$ Encontro Nacional de Química Orgânica $\left(5^{\circ} \mathrm{ENQO}\right)$ irá realizar-se na Aula Magna da Universidade de Aveiro, de 1 a 4 de Julho/2003. Esta reunião de grande significado para cientistas portugueses, principalmente aqueles com interesses científicos centrados na Química Orgânica, contará com a presença de alguns colegas estrangeiros.

No seguimento de contactos havidos com colegas espanhóis e japoneses surgiu a possibilidade de se iniciar num futuro próximo congressos Luso-EspanoJaponeses de Química Orgânica. Como acção preliminar, em tal contexto, e dada a proximidade entre as datas dos encontros Espano-Japonês e do $5^{\circ}$ ENQO, foi decidido incluir no próximo ENQO um simpósio Luso-Japonês a ter lugar no dia 4 de Julho.

0 programa dos primeiros dois dias e meio do $5^{\circ}$ ENQO consistirá em 7 lições plenárias, 14 comunicações orais convidadas e em comunicações em cartaz. A atribuição de 5 das referidas comunicações orais será feita por convite, sendo resultante da análise a fazer aos resumos submetidos. 0 dia 4 de Julho, dia da acção Luso-Japonesa, contará com 5 lições plenárias de cientistas japoneses e com 3 de cientistas portugueses. A listagem dos conferencistas está indicada
Foi Representante Nacional na Working Party on Chemical Education da FECS (Federation of European Chemical Societies) e no Comité Para o Ensino da Química (CTC) da IUPAC.
A morte prematura de Mariana Pereira foi uma perda enorme para a comunidade científica e, em particular, para a Educação em Química em Portugal. em seguida. A circular com todas as indicações do congresso, incluindo o título das conferências, será enviada a todos os sócios da Sociedade Portuguesa de Química, divisão de Química Orgânica, em meados de Março. A data limite do envio de resumos será 15 de Maio. Os preços de inscrição serão os seguintes: Sócios-160 ; não sócios - 210 , estudantes de licenciatura -80 .

\section{Contactos:}

Prof. Doutor Artur M. S. Silva, arturs@dq.ua.pt

Doutora Teresa Pinho e Melo,

tmelo@ci.uc.pt

\section{Lições plenárias:}

- Ana Maria Oliveira Campos, Universidade do Minho

- Anake Kijjoa, Instituto de Ciências Biomédicas de Abel Salazar, Universidade do Porto

- Daniel Dauzonne, Institut Curie, Orsay - Paris (França) (a confirmar)

- R. Alan Aitken, University of St. Andrews, St. Andrews (Reino Unido)

- Steve G. Davies, Oxford University, Oxford (Reino Unido) (a confirmar)

- Sultan T. Abu-Orabi, Yarmouk University, Irbid (Jordânia)

- Juan Antonio Hueso, GlaxoSmithKline, Madrid (Espanha) (a confirmar)

\section{Lições do simpósio Luso-Japonês:}

- Amélia Pilar Rauter, Universidade de Lisboa

- Ana Maria Lobo, Universidade Nova de Lisboa

- José A. S. Cavaleiro, Universidade de Aveiro

- Shun-Ichi Murahashi, Okayama University of Science

- Takeshi Nakai, Niigata University,

- Tamio Hayashi, Kyoto University

- Tamejiro Hiyama, Kyoto University

- Tetsuo Shiba, Osaka University

Comunicações orais Convidadas:

- Albertino Figueiredo, Universidade da Beira Interior

- Amadeu Brigas, Universidade do Algarve

- Arménio Serra, Universidade Católica Portuguesa - Pólo da Figueira da Foz

- Carlos M. Afonso, Universidade Nova de Lisboa

- Ivan Villax, Hovione SA, Loures

- Luís Carvalho, Universidade de Trásos-Montes e Alto Douro

- Mário Q. Simões, Universidade de Aveiro

- Pedro Brito Correia, Herbex, Sintra

- Victor Freitas, Universidade do Porto

\section{Tabela Periódica em CD}

Está a ser distribuída gratuitamente aos sócios uma Tabela Periódica em CD, WinPerio 2.0, produzida pela SPQ. Se já efectuou o pagamento da sua quota de 2003, deve ter recebido o CD juntamente com o recibo do pagamento. Em breve irá também receber o seu novo cartão de sócio.

\section{Contactos da SPQ}

Recorda-se que todos os assuntos relacionados com a SPQ podem ser tratados enviando uma mensagem para o endereço spq@spq.pt. 
Encontros da SPQ em 2003

\begin{tabular}{|c|c|c|c|c|c|}
\hline Data & Nome do Encontro & Local & $\begin{array}{l}\text { Pres. Comissão } \\
\text { Organizadora }\end{array}$ & $\begin{array}{l}\text { Contacto } \\
\text { organização }\end{array}$ & $\begin{array}{l}\text { Página } \\
\text { web }\end{array}$ \\
\hline $\begin{array}{l}30-31 \\
\text { Maio }\end{array}$ & $\begin{array}{l}\text { Catálise e } \\
\text { Materiais Porosos }\end{array}$ & Évora (UE) & Peter Carrott & peter@uevora.pt & $\begin{array}{l}\text { http://www.cqe. } \\
\text { uevora.pt/CMP6 }\end{array}$ \\
\hline $\begin{array}{l}22-25 \\
\text { Junho }\end{array}$ & Química de Alimentos & Lisboa (IPIMAR) & Narcisa Bandarra & narcisa@ipimar.pt & $\begin{array}{l}\text { http://ipimar-iniap. } \\
\text { ipimar.pt/6eqa }\end{array}$ \\
\hline $\begin{array}{l}1-4 \\
\text { Julho }\end{array}$ & Química Orgânica & Aveiro (UA) & Artur Silva & arturs@dq.ua.pt & tmelo@ci.uc.pt \\
\hline $\begin{array}{l}31 \text { Agosto } \\
3 \text { Setembro }\end{array}$ & Química-Física & Lisboa (FCUL) & $\begin{array}{l}\text { Fernando } \\
\text { Fernandes }\end{array}$ & 6eqf-spq@fc.ul.pt & $\begin{array}{l}\text { http://6eqf } \\
\text {-spq.fc.ul.pt/ }\end{array}$ \\
\hline $\begin{array}{l}7-10 \\
\text { Setembro }\end{array}$ & Glúcidos (Glupor 5) & Covilhã (UBI) & M. Isabel Ismael & glupor5@ubista.ubi.pt & $\begin{array}{l}\text { http://www. } \\
\text { ubi.pt/glupor5/ }\end{array}$ \\
\hline $\begin{array}{l}15-20 \\
\text { Setembro }\end{array}$ & Fotoquímica & $\begin{array}{l}\text { S. de Compostela } \\
\text { (Espanha) }\end{array}$ & Flor R. Prieto & jifotoq@usc.es & $\begin{array}{l}\text { http://www.usc. } \\
\text { es/jifotoq/pt/ }\end{array}$ \\
\hline $\begin{array}{l}\text { 17-18 } \\
\text { Outubro }\end{array}$ & Química Industrial & Sintra & J. Costa Reis & petrolabe@netc.pt & \\
\hline $\begin{array}{l}18-19 \\
\text { Novembro }\end{array}$ & Química Analítica & Porto & J. Costa Lima & limajlfc@ff.up.pt & \\
\hline $\begin{array}{l}20-21 \\
\text { Novembro }\end{array}$ & $\begin{array}{l}\text { Ensino e Divulgação } \\
\text { da Química }\end{array}$ & Porto & $\begin{array}{l}\text { João Paiva } \\
\text { Duarte Costa Pereira }\end{array}$ & jcpaiva@netcabo.pt & http://www.spq.pt \\
\hline $\begin{array}{l}15-17 \\
\text { Dezembro }\end{array}$ & $\begin{array}{l}\text { Nacional de } \\
\text { Cromatografia }\end{array}$ & Lisboa(T. Tombo) & J. M. F. Nogueira & nogueira@fc.ul.pt & $\begin{array}{l}\text { http://www.spq. } \\
\text { pt/cromallI }\end{array}$ \\
\hline
\end{tabular}

Não se realiza enc. separado de Q. Inorgânica. Coincide com:

- FIGIPS7, 11-14 Junho 2003, FCUL, Lisboa. (http://www.figips7.fc.ul.pt/)

Também apoiado pela SPQ:

- In Vino Analytica Scientia III, 10-12 Julho 2003, Aveiro.(http://www.dq.ua.pt/invino/)

- $2^{\circ}$ Encontro Luso-Brasileiro de RMN, 23-26 Setembro 2003, Sintra. (http///www.dq.fct.unl.pt/ELBRMN-Portugal/)

\section{Ainda os 90 anos da SPQ}

Por razões várias, a lista dos sócios publicada no anterior Química estava incompleta. Publicam-se agora os nomes de mais 83 sócios. Destes, alguns são novos, ou actualizaram entretanto as suas quotas. Outros, não foram publicados por deficiências da base de dados, agora inteiramente refeita. Aos sócios lesados as nossas sinceras desculpas.

Abel Gomes Martins Ferreira

Abel José de Sousa Costa Vieira

Adelaide Gabriel de Oliveira Baptista

Aluisio da Cruz Marques Leal

Amélia Maria Pina Soares Gonçalves da Silva Ana Cristina Hurtado de Matos Coelho Ana Filipa Pinto Godinho

Ana Isabel Ramos N. Amorim Barros Ana Maria da Cruz D. M. de Castro Galvão
Ana Maria dos Santos Rosa da Costa Ana Maria Félix Trindade Lobo Ana Maria Melo Ventura Reis Ana Maria Vieira da Silva Viana Cavaleiro Ana Paula da Silva Correia Ana Paula da Silva Marques da Cunha CarvaIho

Ana Verina Faria Fernandes

Carla Isabel Costa Pinheiro

Carla Maria Duarte Nunes

Carlos Jorge Ramcs Rebelo dos Santos

Catarina Isabel da Silva Ralha

Christopher David Maycock

Cristina Maria Ascenção Alonso

Cristina Maria Ramalho Ferreira Barros

Dina Isabel Malheiros Dinis de Mendonça

Dora Sofia Ferreira Dias Santos

Elisabete Maria dos S. Castanheira Coutinho

Fernando Manuel Sebastião Silva Fernandes

Filomena Elisabete Lopes Martins Elvas Leitão
Guida Maria Martins de Bastos Helena Isabel Seguro Nogueira João Sérgio Seixas de Melo Joaquim José Azevedo Moura Ramos Jorge Manuel Dias Guerreiro

José Manuel da Cunha Oliveira Figueira Carretas

Lara Isabel Penedo Lança P. Pellegrino Luís Miguel Silva da Cruz Luisa Margarida Dias Ribeiro de Sousa Martins

Luiza Margarida Gehl da Fonseca Bastos B Melo

Margarida Maia Rebelo V. Ferreira

Maria Augusta dos Santos Leitão Rodrigues Gomes

Maria Cândida dos Remédios Barradas Rodrigues

Maria Cidália Ferreira da Torre Abreu Maria Clara Ramalho Monteiro Pires Basto 


\section{Maria Cristina de Oliveira da Costa}

Maria Cristina Froes Brilhante D. Gomes Azevedo

Maria da Conceição Fontes de Sá O. Lopes Dias

Maria da Soledade Costa Cravo da Silva San-

tos

Maria de Fátima Candeias Coelho Teixeira

Maria de Fátima de Oliveira

Maria de Fátima Monteiro Martins Minas da Piedade

Maria de Lurdes dos Santos Cristiano

Maria de Lurdes Taveira Sadler Simões Gonçalves

Maria do Pilar Figueroa Gonçalves

Maria do Rosário Gonçalves Reis Marques
Maria Helena de C. Borges Lencart e Silva

Maria Helena Freitas Casimiro

Maria Isabel Pinto dos Reis

Maria João Pereira Marques Paz Melo de Carvalho

Maria João Pinto da Cruz Fernandes de Lemos

Maria João Raposo da Silva Figueira

Maria José Cambalacho Rosa Mendes Diogo

Maria José Mendes de Sousa

Maria Lúcia Almeida da Silva

Maria Lurdes Leal Monteiro

Maria Manuel Silva Oliveira

Maria Manuela Afonso da Cruz Correia Bandarra

Maria Manuela Lopes Ribeiro Carrott

Maria Marise Simões de Almeida
Maria Nubélia Silvestre Bravo

Maria Paula Alves Robalo

Maria Teresa Barros da Silva

Maria Teresa Ribeiro de Lima

Maria Teresa S. Pessoa de Amorim

Miguel Cândido Almeida Reis Ribeiro

Nídia Maria Dias Azinheira Rebelo Braz

Paula Alexandra de Carvalho Gomes

Paula Alexandra Pires Esperança

Paula Maria Soares Romão

Paulo Aloísio Edmond Reis da Silva Augusto Rita Virgínia Delgado António

Ruy Eugénio Carvalho Pinto

Susana Maria de Almeida Cardoso

Tito da Silva Trindade

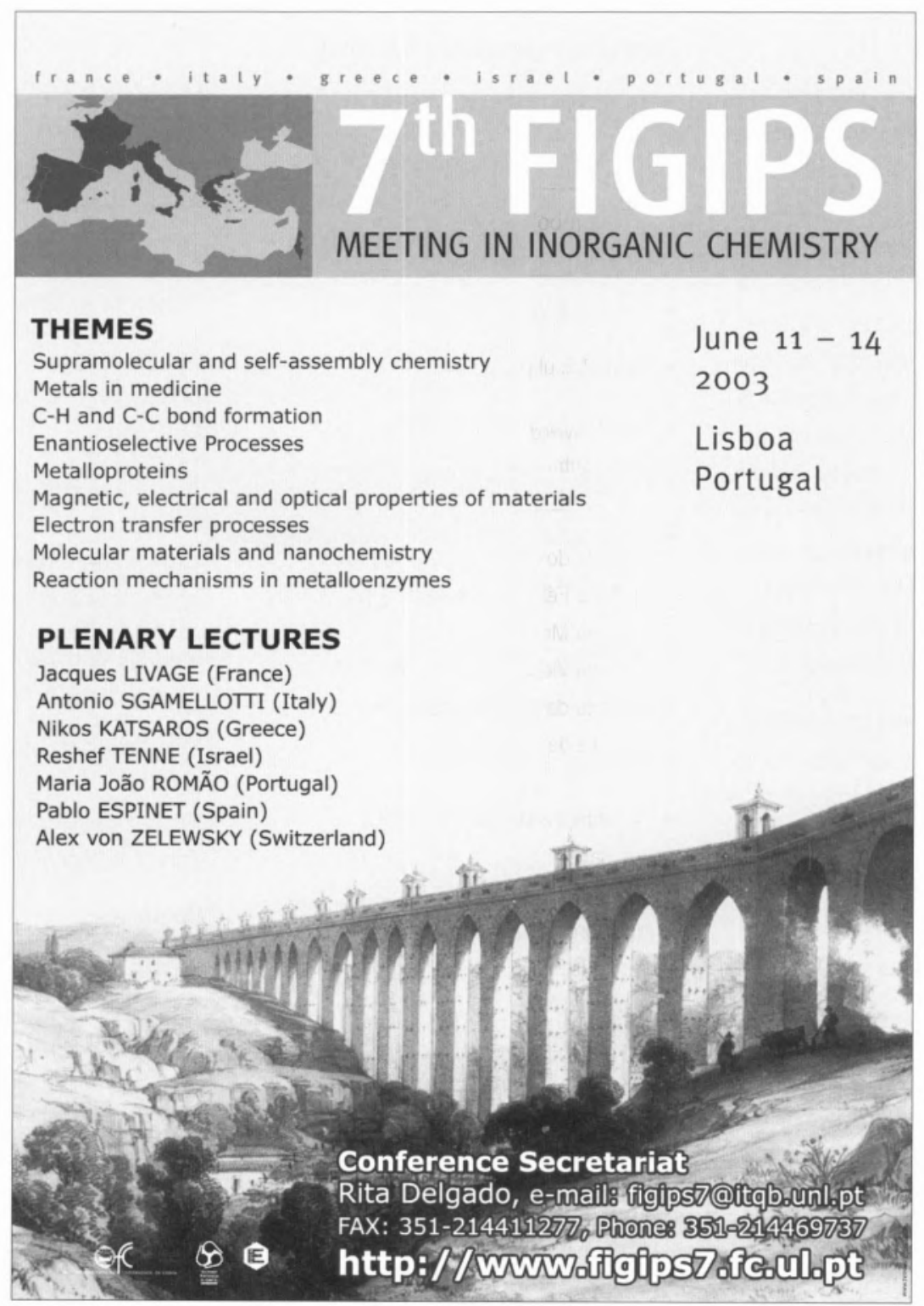

